Shalabh, Toutenburg:

Consequences of Departure from Normality on the Properties of Calibration Estimators

Sonderforschungsbereich 386, Paper 441 (2005)

Online unter: http://epub.ub.uni-muenchen.de/

Projektpartner
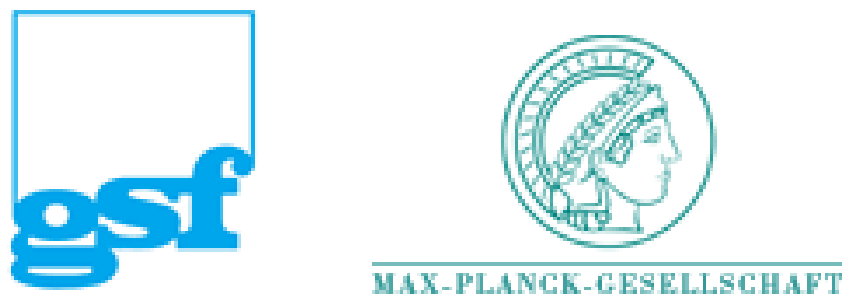


\title{
Consequences of Departure from Normality on the Properties of Calibration Estimators
}

\author{
Shalabh * $^{*} \quad$ H. Toutenburg ${ }^{\dagger}$
}

May 24, 2005

\begin{abstract}
This paper considers the classical and inverse calibration estimators and discusses the consequences of departure from normality of errors on their bias and mean squared error properties when the errors in calibration process are small.
\end{abstract}

\section{Introduction}

The problem of calibration in the linear regression analysis deals with the problem of determination of value of an independent variable corresponding to a given value of dependent variable. Generally, the direct or classical regression and inverse regression techniques are employed to obtain the calibration estimators. This has received attention in the literature from various aspects, e.g., Krutchkoff $(1967,1969)$ considered the classical and inverse calibration estimators and concluded through Monte-Carlo simulation that inverse calibration approach is better than classical calibration from a mean squared error point of view in the range of calibration and has larger mean squared error in extrapolation. Later, Halperin (1970) (see also, Williams (1969)) concluded that classical calibration estimator is superior even inside the range of calibration. Krutchkoff (1971) again addressed the issue under Pitman closeness criterion and concluded through simulation for inverse calibration estimator to be still superior or equivalent to classical calibration estimator. The range where classical calibration estimator is superior, it is only mildly superior. Beside these debates over the superiority of classical and inverse calibration estimators, Tallis (1969) discussed about the aspect of theory of identifiability of mixtures of distribution whereas Pepper (1973) considered the problem of calibration in the light of random walk. The calibration problem in the bayesian framework was considered by Dunsmore (1968), Williford, Carter and Field (1979), and, Hunter and Lamboy (1981). Later, Scheffe (1973) published a detailed work on calibration. The multivariate variant of calibration problem was considered by Friedland (1977), Brown (1982) with discussion and Brown and Sundberg (1987, 1989). Some other aspects of calibration problem are dealt in Berkson (1969), Minder and Whitney (1975), Aitchison (1977), Lwin and Maritz( 1980), Lwin and Spiegelmann (1986), and Misquitta and Ruymgaart $\left(200^{*}\right)$.

The performance properties of the classical and inverse calibration estimators along with their modified and extended forms have been extensively investigated under the normality of errors; see, e.g., Osborne (1991) for an interesting review. Also, Brown (1993, Chap. 2) discusses the aspect of controlled calibration with classical and inverse calibration estimators but under the assumption of normal distribution of errors. What happens when the distribution of errors depart from normality has been treated neglectfully in the literature. This communication reports a modest effort in this direction. For example, Lwin and

\footnotetext{
* Department of Mathematics and Statistics, Indian Institute of Technology, Kanpur, India, Email: shalab@iitk.ac.in; shalabh1@yahoo.com

${ }^{\dagger}$ Institut für Statistik, Universität München, 80799 München, Germany, Email: Helge.Toutenburg@stat.uni-muenchen.de
} 
Spiegelmann (1986) considered a carbon dating example under the assumption of normally distributed errors. If disturbances do not necessarily follow a normal distribution, then it is not clear from this example that how the efficiency properties are changed.

If a functional form of the distribution of errors is assumed, it may not be difficult to analyze the performance of calibration estimators but then the inferences drawn will be valid for that specific distribution and consequently no general prescription can be given to practitioners. Such is, however, not the case if we deal with the asymptotic properties of the calibration estimators. For this purpose, following Srivastava and Singh (1989), we employ the small error asymptotic theory. A distinguishing feature of the small error asymptotic theory is that it rests upon the assumption that errors in the calibration process are small which is reasonable as well as tenable because calibration experiments are usually conducted in a controlled environment and every precaution is exercised to reduce the errors as far as possible in a bid to accomplish a high quality level of the instrument. Clearly, an instrument giving imprecise and inaccurate results has little utility and many people will be unwilling to use it. Further, the small error asymptotic theory does not require the number of observations in the calibration process to be sufficiently large as is the case with the large sample asymptotic theory.

The organization of our presentation is as follows. In Section 2, we describe the framework and present the classical and inverse calibration estimators. Their bias properties are analyzed in Section 3 and the effect of departure from the normality of errors is studied. Similarly, their mean squared error properties are discussed in Section 4. An illustrative example is provided in Section 5 and some concluding remarks are offered in Section 6 . Lastly, the Appendix provides proofs of Theorems.

\section{The Calibration Estimators}

Let us consider a simple calibration experiment in which $y_{1}, y_{2}, \ldots, y_{n}$ refer to the responses or readings on the instrument corresponding to $n$ known values $x_{1}, x_{2}, \ldots, x_{n}$. Further, let $Y$ be the response or reading on the instrument corresponding to an unknown value $X$.

Assuming the response relationship to be linear, we can write

$$
\begin{aligned}
& y_{i}=\alpha+\beta x_{i}+u_{i} \quad(i=1,2, \ldots, n) \\
& Y=\alpha+\beta X+U
\end{aligned}
$$

where $\alpha$ is the intercept term, $\beta$ is the slope parameter and $u_{1}, u_{2}, \ldots, u_{n}, U$ are the errors.

Let us define

$$
\begin{aligned}
\bar{x}=\frac{1}{n} \sum x_{i}, \quad s_{x}^{2} & =\frac{1}{n} \sum\left(x_{i}-\bar{x}\right)^{2}, \\
\bar{y}=\frac{1}{n} \sum y_{i}, \quad s_{y}^{2} & =\frac{1}{n} \sum\left(y_{i}-\bar{y}\right)^{2}, \\
s_{x y} & =\frac{1}{n} \sum\left(x_{i}-\bar{x}\right)\left(y_{i}-\bar{y}\right) .
\end{aligned}
$$

For the statistical estimation of unknown $X$, there are two approaches. One is the classical approach in which the regression line of $y_{i}$ on $x_{i}$ is used to formulate an estimator of $X$ :

$$
\hat{X}_{C}=\bar{x}+\frac{s_{x}^{2}}{s_{x y}}(Y-\bar{y})
$$

which is the classical calibration estimator (CCE).

The other approach is based on inverse regression in which the regression line of $x_{i}$ on $y_{i}$ is utilized to formulate the following estimator of $X$ :

$$
\hat{X}_{I}=\bar{x}+\frac{s_{x y}}{s_{y}^{2}}(Y-\bar{y})
$$


which is known as the inverse calibration estimator (ICE).

For analyzing the performance properties of the calibration estimators (2.4) and (2.5), we do not assume any specific distribution like the normality of errors in the regression model. All that is supposed is the finiteness of moments up to order four. Accordingly, let the first four moments of the independently and identically distributed errors $u_{1}, u_{2}, \ldots, u_{n}, U$ be $0, \sigma^{2}, \sigma^{3} \gamma_{1}$ and $\sigma^{4}\left(\gamma_{2}+3\right)$ so that the quantities $\gamma_{1}$ and $\gamma_{2}$ are the Pearson's measures of skewness and kurtosis respectively.

\section{Comparison under Bias Criterion}

Utilizing the small errors asymptotic theory, it is easy to see that both the calibration estimators $\hat{X}_{C}$ and $\hat{X}_{I}$ are consistent but generally biased. The asymptotic approximations for the biases of $\hat{X}_{C}$ and $\hat{X}_{I}$ are obtained by Srivastava and Singh (1989) under the assumption that the errors follow a normal distribution. Their expressions, however, remain unaltered even when the distribution is not normal. We reproduce them for the sake of ready reference:

$$
\begin{aligned}
B\left(\hat{X}_{C}\right) & =E\left(\hat{X}_{C}-X\right) \\
& =-\frac{\sigma^{2} d}{n \beta^{2} s_{x}} \\
B\left(\hat{X}_{I}\right) & =E\left(\hat{X}_{I}-X\right) \\
& =\frac{\sigma^{2} d(n-3)}{n \beta^{2} s_{x}}
\end{aligned}
$$

to order $O\left(\sigma^{2}\right)$ where

$$
d=\left(\frac{\bar{x}-X}{s_{x}}\right)
$$

As $n=2$ is the trivial case in which the two regression lines coincide, we restrict our attention to $n>2$. If $n=3$, we observe from (3.2) that the ICE is almost or nearly unbiased in the sense that bias to the order of our approximation vanishes. If $n>3$, both CCE and ICE are biased but in opposite directions. However, the CCE has smaller magnitude of bias in comparison to the ICE when $n$ is greater than 4 .

In order to study the effect of non-normal error distributions, we need to consider the higher order approximations.

Theorem 3.1:

If the error distribution is asymmetric, i.e., $\gamma_{1} \neq 0$, the asymptotic approximations for the biases to order $O\left(\sigma^{3}\right)$ are given by

$$
\begin{aligned}
B\left(\hat{X}_{C}\right) & =-\frac{\sigma^{2} d}{n \beta^{2} s_{x}}+\frac{\sigma^{3} \gamma_{1}}{n^{2} \beta^{3} s_{x}^{2}}\left[d \gamma_{1 x}-1\right] \\
B\left(\hat{X}_{I}\right) & =\frac{\sigma^{2} d(n-3)}{n \beta^{2} s_{x}}+\frac{\sigma^{3} \gamma_{1}}{n^{2} \beta^{3} s_{x}^{2}}\left[4 d \gamma_{1 x}+(n-3)\right]
\end{aligned}
$$

where

$$
\gamma_{1 x}=\frac{1}{s_{x}^{3} n} \sum^{n}\left(x_{i}-\bar{x}\right)^{3}
$$

Proof:

See Appendix. 
The expressions (3.4) and (3.5) may furnish an idea about the change in bias when the distribution of errors is skewed. For instance, suppose that $\gamma_{1}$ and $\beta$ have same signs, i.e., the slope parameter $\beta$ is positive for positively skewed distribution and is negative for negatively skewed distribution. Now if $d>0$ (i.e., the unknown value $X$ is below the average $\bar{x}$ of the chosen values in the calibration process) and $\gamma>\left(\frac{1}{d}\right)$, the contribution of the term of order $O\left(\sigma^{3}\right)$ will tend to lower the magnitude of bias of the CCE while it will lead to an increase in the bias of the ICE. The opposite is true when $\gamma_{1}$ and $\beta$ have opposite signs.

When the distribution of errors is symmetric, the contribution of the term of order $O\left(\sigma^{3}\right)$ vanishes. In order to further examine the effect of the departure from normality on the bias, let us consider the bias expressions to a higher order of approximation.

\section{Theorem 3.2:}

If the error distribution is symmetric, the asymptotic approximations for the biases to order $O\left(\sigma^{4}\right)$ are given by

$$
\begin{aligned}
B\left(\hat{X}_{C}\right) & =-\frac{\sigma^{2} d}{n \beta^{2} s_{x}}-\frac{\sigma^{4}}{n^{3} \beta^{4} s_{x}^{3}}\left[3 n d+\gamma_{2} T_{C}\right] \\
B\left(\hat{X}_{I}\right) & =\frac{\sigma^{2} d(n-3)}{n \beta^{2} s_{x}}-\frac{\sigma^{4}}{n^{3} \beta^{4} s_{x}^{3}}\left[(n-3)(n-5) n d+\gamma_{2} T_{I}\right]
\end{aligned}
$$

where

$$
\begin{aligned}
T_{C} & =\left(3+\gamma_{2 x}\right) d-\gamma_{1 x} \\
T_{I} & =\left(n^{2}-10 n+33+\gamma_{2 x}\right) d-4 \gamma_{1 x}
\end{aligned}
$$

with

$$
\gamma_{2 x}=\frac{1}{n s_{x}^{4}} \sum\left(x_{i}-\bar{x}\right)^{4}-3
$$

$\underline{\text { Proof: }}$

See Appendix.

It is interesting to observe from (3.7) and (3.8) that the contribution of the term of order $O\left(\sigma^{4}\right)$ increases the magnitude of the bias of the CCE when the distribution of errors is mesokurtic or normal. In case of the ICE, this term decreases the bias provided that $n>5$. These results may not be necessarily true when distribution is different from normal. Besides the peakedness of the error distribution, the contribution now additionally depends upon the skewness and kurtosis of the chosen values $x_{1}, x_{2}, \ldots, x_{n}$; see the expressions (3.9) and $(3.10)$.

\section{Comparison under Mean Squared Error Criterion}

If we compare the calibration estimators under the criterion of mean squared error to order $O\left(\sigma^{2}\right)$, both the CCE and ICE are found to have same mean squared error $\sigma^{2} \Omega$ where

$$
\Omega=\left(\frac{d^{2}+n+1}{n \beta^{2}}\right) .
$$

We therefore need to consider higher order approximations of the mean squared errors in order to discriminate the calibration estimators. 


\subsection{Asymmetric Error Distribution Case}

Let us first assume that the distribution of errors is not symmetric.

Theorem 4.1:

The asymptotic approximations for the mean squared errors of the calibration estimators to order $O\left(\sigma^{3}\right)$ are given by

$$
\begin{aligned}
M\left(\hat{X}_{C}\right) & =E\left(\hat{X}_{C}-X\right)^{2} \\
& =\sigma^{2} \Omega-\frac{2 \sigma^{3} \gamma_{1} d}{n^{2} \beta^{3} s_{x}^{2}}\left(d \gamma_{1 x}-2\right) \\
M\left(\hat{X}_{I}\right) & =E\left(\hat{X}_{I}-X\right)^{2} \\
& =\sigma^{2} \Omega-\frac{2 \sigma^{3} \gamma_{1} d}{n^{2} \beta^{3} s_{x}^{2}}\left(2 d \gamma_{1 x}-4+n\right)
\end{aligned}
$$

Proof:

See Appendix.

From (4.2) and (4.3), we observe that

$$
M\left(\hat{X}_{C}\right)-M\left(\hat{X}_{I}\right)=\frac{2 \sigma^{3} q}{n^{2} \beta^{2} s_{x}^{2}}
$$

where

$$
q=\frac{\gamma_{1} d}{\beta}\left(d \gamma_{1 x}-2+n\right)
$$

It is interesting to note that both the CCE and ICE have identical performance with respect to the criterion of mean squared error to order $O\left(\sigma^{3}\right)$ when the distribution of error is symmetric with normal as a particular case. This result does not remain true in general when the distribution of error is skewed or is different from normal. Further, we observe that the CCE is superior (inferior) to the ICE according as the quantity $q$ is negative (positive).

We thus find that the superiority of one calibration estimator over the other hinges upon the sign of $q$ which depends upon $\beta$ (the slope parameter), $\gamma_{1}$ (the skewness coefficient of the error distribution), $\gamma_{1 x}$ (the skewness coefficient of the chosen standard values $x_{1}, x_{2}, \ldots, x_{n}$ ), $\mathrm{d}$ (the difference between the unknown value $X$ and the mean of standard values $x_{1}, x_{2}, \ldots, x_{n}$ ) and $n$ (the number of observations in the calibration experiment).

Let us first assume that $\gamma_{1}$ and $\beta$ have same signs. Then the quantity $q$ is positive when one of the following conditions holds:

$$
\begin{array}{ll}
d \gamma_{1 x}>-(n-2) & \text { if } d>0 \\
d \gamma_{1 x}<-(n-2) & \text { if } d<0 .
\end{array}
$$

When the unknown value $X$ is below $\bar{x}$, the condition (4.6) always holds so long as $\gamma_{1 x}$ or the third central moment of $x_{1}, x_{2}, \ldots, x_{n}$ is positive. If $\gamma_{1 x}$ is negative, the condition (4.6) is satisfied provided that the magnitude of $\gamma_{1 x}$ is less than $(n-2)$. Similarly, when the unknown value $X$ is above the average $\bar{x}$, the condition (4.7) holds only for positive values of $\gamma_{1 x}$ exceeding the ratio of $(n-2)$ to the absolute value of $d$.

If $\gamma_{1}$ and $\beta$ have opposite signs, the quantity $q$ is positive under either of the following two conditions

$$
\begin{array}{ll}
d \gamma_{1 x}<-(n-2) & \text { if } d>0 \\
d \gamma_{1 x}>-(n-2) & \text { if } d<0
\end{array}
$$


When the unknown value $X$ is below $\bar{x}$, the condition (4.8) is satisfied provided that $\gamma_{1 x}$ is negative and its magnitude is larger than the ratio $\frac{(n-2)}{d}$. On the other hand, when $X$ is above the average $\bar{x}$, the condition (4.9) is always true for all the negative values of $\gamma_{1 x}$. This condition (4.9) is also satisfied for some positive values of $\gamma_{1 x}$ provided that the value of $\gamma_{1 x}$ does not exceed the ratio of $(n-2)$ to the magnitude of $d$.

Likewise, we can identify the situations where the quantity $q$ is negative.

\subsection{Symmetric Error Distribution Case}

Let us now consider the mean squared errors to order $O\left(\sigma^{4}\right)$ when the distribution of errors is symmetric.

\section{Theorem 4.2:}

The asymptotic approximations for the mean squared errors of the calibration estimators to order $O\left(\sigma^{4}\right)$ are given by

$$
\begin{aligned}
M\left(\hat{X}_{C}\right) & =E\left(\hat{X}_{C}-X\right)^{2} \\
& =\sigma^{2} \Omega-\frac{\sigma^{4}}{n^{2} \beta^{4} s_{x}^{2}}\left[9 d^{2}+3(n+1)+\gamma_{2} \Delta_{i}\right] \\
M\left(\hat{X}_{I}\right) & =E\left(\hat{X}_{I}-X\right)^{2} \\
& =\sigma^{2} \Omega-\frac{\sigma^{4}}{n^{2} \beta^{4} s_{x}^{2}}\left[(n-5)^{2} d^{2}-(2 n-7)(n+1)+\gamma_{2} \Delta_{I}\right]
\end{aligned}
$$

where

$$
\begin{aligned}
\Delta_{i} & =\frac{3}{n}\left[1-2 d \gamma_{1 x}+\left(3+\gamma_{2 x}\right) d^{2}\right] \\
\Delta_{I} & =\frac{1}{n}\left[7-2 n-16 d \gamma_{1 x}+\left(n^{2}-12 n+47+12 \gamma_{2 x}\right) d^{2}\right]
\end{aligned}
$$

\section{Proof:}

See Appendix.

From (4.10) and (4.11), we observe that

$$
\begin{aligned}
M\left(\hat{X}_{C}\right)-M\left(\hat{X}_{I}\right)= & \frac{\sigma^{4}}{n^{2} \beta^{4} s_{x}^{2}}\left[(n-2)(8-n) d^{2}\right. \\
& \left.+2(n-2)(n+1)+\gamma_{2}\left(\Delta_{i}-\Delta_{I}\right)\right]
\end{aligned}
$$

from which it follows that the ICE is superior to the CCE when

$$
\left[(8-n) d^{2}+2(n+1)\right]>\left(\frac{\gamma_{2}}{n-2}\right)\left(\Delta_{I}-\Delta_{i}\right) .
$$

When the distribution of errors is mesokurtic $\left(\gamma_{2}=0\right)$ or normal, the condition (4.15) holds so long as $n \leq 8$. This condition also holds for $n>8$ provided that

$$
d^{2}<2\left(\frac{n+1}{n-8}\right)
$$

which is satisfied as long as $d^{2}$ is less than 2 .

On the other hand, the CCE has better performance than the ICE in case of $\gamma_{2}=0$ when the inequality (4.16) holds with reversed sign.

The superiority of one calibration estimator over the other under mesokurtic or normal errors, it may be appreciated from (4.15) may not necessarily carry over to the cases of non-normal errors. 


\section{An Illustrative Example}

Let us consider the data related to the pressure in a hydrodynamic system presented in Dunsmore (1968). The pressure is measured at two points yielding the following observations:

$$
\begin{array}{lllllll}
x: & 2.38, & 3.89, & 4.60, & 5.36, & 6.50, & 7.45, \\
y: & 2.70, & 4.01, & 4.41, & 6.00, & 6.05, & 7.41, \\
& & & & \\
x: & 7.81, & 8.38, & 9.09, & 9.89, & 10.58, & 10.98 \\
y: & 8.58, & 8.49, & 9.99, & 9.80, & 10.33, & 11.20
\end{array} .
$$

As the instrument yielding the $x$ values were not available later on, only the $y$ values could be recorded in future. Thus, given a value $Y$, we are to estimate the corresponding $X$.

From the available twelve pairs of observations, we have

$$
\begin{aligned}
& \bar{x}=7.2425, \quad s_{x}^{2}=6.9292, \\
& \gamma_{1 x}=-0.2845, \quad \gamma_{2 x}=-1.0411 \text {. }
\end{aligned}
$$

Using these values in (3.4) and (3.5), we find the bias expressions to order $O\left(\sigma^{3}\right)$ are

$$
\begin{aligned}
& B\left(\hat{X}_{C}\right)=-0.0317\left(\frac{\sigma^{2} d}{\beta^{2}}\right)-(0.0003 d+0.0010)\left(\frac{\sigma^{3} \gamma_{1}}{\beta^{3}}\right) \\
& B\left(\hat{X}_{I}\right)=0.2849\left(\frac{\sigma^{2} d}{\beta^{2}}\right)-(0.0011 d+0.0090)\left(\frac{\sigma^{3} \gamma_{1}}{\beta^{3}}\right)
\end{aligned}
$$

where

$$
d=2.7514-0.3799 X
$$

Similarly, from (3.7) and (3.8), we have

$$
\begin{aligned}
& B\left(\hat{X}_{C}\right)=-0.0317\left(\frac{\sigma^{2} d}{\beta^{2}}\right)-\left[\left(0.0011+1.9589 \gamma_{2}\right) d+0.2845 \gamma_{2}\right]\left(\frac{\sigma}{\beta}\right)^{4} \\
& B\left(\hat{X}_{I}\right)=-0.2849\left(\frac{\sigma^{2} d}{\beta^{2}}\right)-[(0.0320+55.9589) d+1.1380]\left(\frac{\sigma}{\beta}\right)^{4}
\end{aligned}
$$

The expressions (5.1), (5.2), (5.4) and (5.5) clearly bring out that the bias properties of the calibration estimators under the specification of normality of errors may be quite different when the distribution departs from normality.

Next, let us examine the mean squared error properties of the calibration estimators.

When the distribution of errors is asymmetric, the asymptotic approximations of the mean squared errors from (4.1), (4.2) and (4.3) are

$$
\begin{aligned}
& M\left(\hat{X}_{C}\right)=\left(0.833 d^{2}+1.0833\right)\left(\frac{\sigma}{\beta}\right)^{2}+(0.0006 d+0.0040)\left(\frac{\sigma^{3} \gamma_{1} d}{\beta^{3}}\right) \\
& M\left(\hat{X}_{I}\right)=\left(0.833 d^{2}+1.0833\right)\left(\frac{\sigma}{\beta}\right)^{2}+(0.0011 d-0.0160)\left(\frac{\sigma^{3} \gamma_{1} d}{\beta^{3}}\right) .
\end{aligned}
$$

If we look at the twelve observations in the calibration experiment, it can be easily ascertained that $\beta$ is positive. Suppose for a moment that the distribution of errors is positively skewed $\left(\gamma_{1}>0\right)$. Now, for all positive values of $\mathrm{d}$, the asymptotic approximations are given 
by the term involving $\left(\frac{\sigma}{\beta}\right)^{2}$ on the right hand side of (5.6) gives an under-reporting of the mean squared error of the CCE. Such an approximation in case of the ICE, however, gives over-reporting for all negative values of $d$ and positive values of $d$ less than 14.54. In a similar way, we can identify situations where the leading term in the mean squared error expression provides an under-reporting or over-reporting.

Comparing (5.6) and (5.7), we observe that the CCE has smaller mean squared error than the ICE when

$$
\gamma_{1} d(d-40)>0
$$

as $\beta$ is positive.

The condition (5.8) is satisfied for all positively skewed distributions when the unknown value $X$ is larger than 7.24. For negatively skewed distributions, the condition (5.8) holds when $X$ is smaller than 7.24. The opposite is true, i.e., the ICE is better than the CCE when $X<7.24$ for positively skewed distributions or $X>7.24$ for negatively skewed distributions.

When the distribution of errors is symmetric, it follows from (4.10) and (4.11) that the mean squared errors to orders $O\left(\sigma^{4}\right)$ are

$$
\begin{aligned}
M\left(\hat{X}_{C}\right)= & \left(0.833 d^{2}+1.0833\right)\left(\frac{\sigma}{\beta}\right)^{2} \\
& +\left[\left(0.0090+0.0005 \gamma_{2}\right) d^{2}+0.0001 \gamma_{2} d+\left(0.0391+0.00002 \gamma_{2}\right)\right] \\
M\left(\hat{X}_{I}\right)= & \left(0.833 d^{2}+1.0833\right)\left(\frac{\sigma}{\beta}\right)^{2} \\
& +\left[\left(0.0491-0.0029 \gamma_{2}\right) d^{2}+0.0004 \gamma_{2} d\right. \\
& \left.-\left(0.2215+0.0014 \gamma_{2}\right)\right]\left(\frac{\sigma}{\beta}\right)^{4}
\end{aligned}
$$

It is obvious from the above expressions that the mean squared error under the normality specification may be quite different when the distribution is not normal.

\section{Some Remarks}

Employing the small error asymptotic theory and assuming the distribution of errors to be not necessarily normal, we have worked out the asymptotic approximations for the biases and mean squared errors of the classical and inverse calibration errors. Interestingly enough, the third and fourth central moment apart from the mean and variance of the chosen standard values $x_{1}, x_{2}, \ldots, x_{n}$ in the process of calibration are found to play an important role in the efficiency properties of the calibration estimators if the distribution of errors is not normal. Their effect disappears totally as soon as normality is assumed. Further, the asymmetry and peakedness of the error distribution are seen to influence the performance of calibration estimators. As compared to the peakedness of the error distribution, the effect of the lack of symmetry is more pronounced. A general conclusion emerging from our investigations is that the relative performance of one calibration estimator over the other under the specification of normality of errors may not necessarily carry over to non-normal distributions and may often be quite jeopardized. 


\section{A Appendix}

For the application of the small error asymptotic theory, we may notice, that $\sigma$ is small and tends to zero when errors are small. Accordingly, we replace $u_{i}$ in (2.1) and $U$ in (2.2) by $\sigma v_{i}$ and $\sigma V$ respectively so that $v_{1}, v_{2}, \ldots, v_{n}, V$ are independently and identically distributed with first four moments as $0,1, \gamma_{1}$ and $\left(\gamma_{2}+3\right)$. Further, we write

$$
\begin{aligned}
\bar{v} & =\frac{1}{n} \sum_{i} v_{i} \\
Z_{1} & =\frac{1}{n \beta s_{x}^{2}} \sum_{i}\left(x_{i}-\bar{x}\right)\left(v_{i}-\bar{v}\right) \\
Z_{2} & =\frac{1}{n \beta s_{x}^{2}} \sum_{i}\left(v_{i}-\bar{v}\right)^{2}
\end{aligned}
$$

so that, from (2.1) and (2.2), we have

$$
\begin{aligned}
(Y-\bar{y}) & =-\beta d s_{x}+\sigma(V-\bar{v}) \\
s_{x y} & =\beta s_{x}^{2}\left(1+\sigma Z_{1}\right) \\
s_{y}^{2} & =\beta^{2} s_{x}^{2}\left(1+2 \sigma Z_{1}+\sigma^{2} Z_{2}\right)
\end{aligned}
$$

where

$$
d=\frac{(\bar{x}-X)}{s_{x}} .
$$

Using these results and following Srivastava and Singh (1989), we can express

$$
\begin{aligned}
\left(\hat{X}_{C}-X\right)= & \sigma\left[d s_{x} Z_{1}+\frac{1}{\beta}(V-\bar{v})\right]\left(1+\sigma Z_{1}\right)^{-1} \\
= & \sigma\left[d s_{x} Z_{1}+\frac{1}{\beta}(V-\bar{v})\right]\left(1-\sigma Z_{1}+\sigma^{2} Z_{1}^{2}-\sigma^{3} Z_{1}^{3}+\ldots\right) \\
= & \sigma\left[d s_{x} Z_{1}+\frac{1}{\beta}(V-\bar{v})\right]-\sigma^{2}\left[d s_{x} Z_{1}^{2}+\frac{1}{\beta}(V-\bar{v}) Z_{1}\right] \\
& +\sigma^{3}\left[d s_{x} Z_{1}^{3}+\frac{1}{\beta}(V-\bar{v}) Z_{1}^{2}\right]-\sigma^{4}\left[d s_{x} Z_{1}^{4}+\frac{1}{\beta}(V-\bar{v}) Z_{1}^{3}\right] \\
& +O_{p}\left(\sigma^{5}\right) .
\end{aligned}
$$

Similarly, it is easy to find that

$$
\begin{aligned}
\left(\hat{X}_{I}-X\right)= & \sigma\left[d s_{x} Z_{1}+\frac{1}{\beta}(V-\bar{v})\right] \\
& -\sigma^{2}\left[d s_{x}\left(2 Z_{1}^{2}-Z_{2}\right)+\frac{1}{\beta}(V-\bar{v}) Z_{1}\right] \\
& +\sigma^{3}\left[d s_{x} Z_{1}\left(4 Z_{1}^{2}-3 Z_{2}\right)+\frac{1}{\beta}(V-\bar{v})\left(2 Z_{1}^{2}-Z_{2}\right)\right] \\
& -\sigma^{4}\left[d s_{x}\left(8 Z_{1}^{4}-8 Z_{1}^{2} Z_{2}+Z_{2}^{2}\right)+\frac{1}{\beta}(V-\bar{v})\left(4 Z_{1}^{2}-3 Z_{2}\right) Z_{1}\right] \\
& +O_{p}\left(\sigma^{4}\right) .
\end{aligned}
$$


Proof of Theorem 3.1:

When the distribution of errors is not symmetric, we observe that

$$
\begin{aligned}
E\left(Z_{1}\right) & =0, \\
E\left(Z_{1}^{2}\right) & =\frac{1}{n \beta^{2} s_{x}^{2}}, \\
E\left(Z_{1}^{3}\right) & =\frac{\gamma_{1} \gamma_{1 x}}{n^{2} \beta^{3} s_{x}^{3}}, \\
E\left(Z_{2}\right) & =\frac{n-1}{n \beta^{2} s_{x}^{2}}, \\
E(V-\bar{v}) & =0, \\
E(V-\bar{v}) Z_{1} & =0, \\
E(V-\bar{v}) Z_{1}^{2} & =-\frac{\gamma_{1}}{n^{2} \beta^{2} s_{x}^{2}}, \\
E(V-\bar{v}) Z_{2} & =-\frac{\gamma_{1}(n-1)}{n^{2} \beta^{2} s_{x}^{2}}, \\
E\left(Z_{1} Z_{2}\right) & =0 .
\end{aligned}
$$

Now the bias expressions to order $O\left(\sigma^{3}\right)$ from (A.1) and (A.2) are

$$
\begin{aligned}
B\left(\hat{X}_{C}\right)= & E\left(\hat{X}_{C}-X\right) \\
= & \sigma\left[d s_{x} E\left(Z_{1}\right)+\frac{1}{\beta} E(V-\bar{v})\right] \\
& -\sigma^{2}\left[d s_{x} E\left(Z_{1}^{2}\right)+\frac{1}{\beta} E(V-\bar{v}) Z_{1}\right] \\
& +\sigma^{3}\left[d s_{x} E\left(Z_{1}^{3}\right)+\frac{1}{\beta} E(V-\bar{v}) Z_{I}^{2}\right] \\
B\left(\hat{X}_{I}\right)= & E\left(\hat{X}_{I}-X\right) \\
= & \sigma\left[d s_{x} E\left(Z_{1}\right)+\frac{1}{\beta} E(V-\bar{v})\right] \\
& -\sigma^{2}\left[d s_{x} E\left(2 Z_{1}^{2}-Z_{2}\right)+\frac{1}{\beta} E(V-\bar{v}) Z_{1}\right] \\
& +\sigma^{3}\left[d s_{x} E\left(4 Z_{1}^{3}-3 Z_{1} Z_{2}\right)+\frac{1}{\beta} E(V-\bar{v})\left(2 Z_{I}^{2}-Z_{2}\right)\right] .
\end{aligned}
$$

Substituting (A.3), we obtain the results stated in Theorem 3.1.

\section{Proof of Theorem 3.2:}

When the distribution of errors is symmetric so that $\gamma_{1}=0$, the expectations of the terms of order $O\left(\sigma^{3}\right)$ in the expressions (A.1) and (A.2) are zero. We therefore need to consider the terms of order $O\left(\sigma^{4}\right)$.

Now it can be easily seen that

$$
\begin{aligned}
E\left(Z_{1}^{4}\right) & =\frac{1}{n^{3} \beta^{4} s_{x}^{4}}\left[3 n+\gamma_{2}\left(3+\gamma_{2 x}\right)\right], \\
E\left(Z_{1}^{2} Z_{2}\right) & =\frac{1}{n^{3} \beta^{4} s_{x}^{4}}\left[n(n+1)+\gamma_{2}(n-1)\right], \\
E\left(Z_{2}^{2}\right) & =\frac{(n-1)}{n^{3} \beta^{4} s_{x}^{4}}\left[n(n+1)+\gamma_{2}(n-1)\right], \\
E(V-\bar{v}) Z_{1}^{3} & =-\frac{\gamma_{2} \gamma_{1 x}}{n^{3} \beta^{3} s_{x}^{3}}, \\
E(V-\bar{v}) Z_{1} Z_{2} & =0
\end{aligned}
$$


Utilizing the results (A.3) and (A.6), the expressions mentioned in Theorem 3.2 can be straightforwardly obtained from (A.1) and (A.2).

\section{Proof of Theorem 4.1:}

First of all, we notice that

$$
\begin{aligned}
E\left[d s_{x} Z_{1}+\frac{1}{\beta}(V-\bar{v})\right]^{2} & =d^{2} s_{x}^{2} E\left(Z_{1}^{2}\right)+\frac{1}{\beta^{2}} E(V-\bar{v})^{2}+\frac{2}{\beta} d s_{x} E(V-\bar{v}) Z_{1} \\
& =\frac{1}{n \beta^{2}}\left(d^{2}+n+1\right) \\
& =\Omega .
\end{aligned}
$$

Now, from (A.1) and (A.2), the mean squared errors to order $O\left(\sigma^{3}\right)$ are

$$
\begin{aligned}
M\left(\hat{X}_{C}\right)= & E\left(\hat{X}_{C}-X\right)^{2} \\
= & \sigma^{2} \Omega-2 \sigma^{3} E\left[d^{2} s_{x}^{2} Z_{1}^{3}+\frac{1}{\beta^{2}}(V-\bar{v})^{2} Z_{1}+\frac{2}{\beta} d s_{x} E(V-\bar{v}) Z_{1}^{2}\right] \\
M\left(\hat{X}_{I}\right)= & E\left(\hat{X}_{I}-X\right)^{2} \\
= & \sigma^{2} \Omega-2 \sigma^{3} E\left[d^{2} s_{x}^{2}\left(2 Z_{1}^{3}-Z_{1} Z_{2}\right)+\frac{1}{\beta^{2}}(V-\bar{v})^{2} Z_{1}\right. \\
& \left.+\frac{1}{\beta} d s_{x}(V-\bar{v})\left(3 Z_{1}^{2}-Z_{2}\right)\right] .
\end{aligned}
$$

Using the results (A.3) along with

$$
E(V-\bar{v})^{2} Z_{1}=0
$$

under the specification of asymmetric error distribution, we find the expressions given in Theorem 4.1.

Proof of Theorem 4.2: As the distribution of errors is now assumed to be symmetric, the terms of order $O\left(\sigma^{3}\right)$ in the expressions for the mean squared errors vanish. Consequently, if we retain the terms of order $O\left(\sigma^{4}\right)$, we have

$$
\begin{aligned}
M\left(\hat{X}_{C}\right)= & \sigma^{2} \Omega+3 \sigma^{2} E\left[d^{2} s_{x}^{2} Z_{1}^{4}+\frac{1}{\beta^{2}}(V-\bar{v})^{2} Z_{1}^{2}+\frac{2}{\beta} d s_{x}(V-\bar{v}) Z_{1}^{3}\right] \\
M\left(\hat{X}_{I}\right)= & \sigma^{2} \Omega+\sigma^{4} E\left[d^{2} s_{x}^{2}\left(12 Z_{1}^{4}-10 Z_{1}^{2} Z_{2}+Z_{2}^{2}\right)\right. \\
& \left.+\frac{1}{\beta^{2}}(V-\bar{v})^{2}\left(5 Z_{1}^{2}-2 Z_{2}\right)+\frac{2}{\beta} d s_{x}(V-\bar{v})\left(8 Z_{1}^{2}-5 Z_{1} Z_{2}\right)\right]
\end{aligned}
$$

Substituting

$$
\begin{aligned}
& E(V-\bar{v})^{2} Z_{1}^{2}=\frac{1}{n^{3} \beta^{2} s_{x}^{2}}\left[n(n+1)+\gamma_{2}\right] \\
& E(V-\bar{v})^{2} Z_{2}=\frac{(n-1)}{n^{3} \beta^{2} s_{x}^{2}}\left[n(n+1)+\gamma_{2}\right]
\end{aligned}
$$

and (A.6) in (A.10) and (A.11), we obtain the results stated in Theorem 4.2. 


\section{References}

Aitchison, John A. (1977): "Calibration problem in statistical diagnosis: the system transfer problem", Biometrika , 64, no. 3, 461-472.

Berkson J. (1969): "Estimation of a linear function for a calibration line: Consideration of a recent proposal", Technometrics, 11, 649-660.

Brown, P. J. (1982): "Multivariate calibration. With discussion", Journal of the Royal Statistical Society, Series B, 44, no. 3, 287-321.

Brown, Philip J. and Rolf Sundberg (1987): "Confidence and conflict in multivariate calibration", Journal of the Royal Statistical Society, Series B, 49, no. $1,46-57$.

Brown, Philip J. and Rolf Sundberg (1989): "Prediction diagnostics and updating in multivariate calibration" Biometrika, 76, no. 2, 349-361.

Brown, Philip J. (1993): Measurement Error, regression and calibration, Oxford Statistical Science Series, The Clarendon Press, Oxford University Press, New York.

Dunsmore, I. R. (1968): "A bayesian approach to calibration", Journal of the Royal Statistical Society, Series B, 30, 396-405.

Friedland, Bernard (1977): "On the calibration problem", IEEE Trans. Automatic Control, AC-22, no. 6, 899-905

Halperin, M. (1970) : "On inverse estimation in linear regression", Technometrics, 12, pp. 727-736

Hunter, William G. and Warren F. Lamboy (1981): "A Bayesian analysis of the linear calibration problem", Technometrics, 23, no. 4, 323-350.

Krutchkoff, R. G. (1967): "Classical and inverse regression methods of calibration", Technometrics, 9, 425-439.

Krutchkoff, R. G. (1969): "Classical and inverse regression methods of calibration in extrapolation", Technometrics, 11, 605-608.

Krutchkoff, Richard G. (1971): "The calibration problem and closeness", J. Statist. comp. and Simulation, 1, no. 1, 87-95.

Lwin, T. and J.S. Maritz (1980): "A note on the problem of statistical calibration", Journal of the Royal Statistical Society, Series C, 29, no. 2, 135-141.

Lwin, T. and Clifford H. Spiegelman (1986): "Calibration with working standards", Journal of the Royal Statistical Society, Series C, 35, no. 3, 256-261.

Minder, Ch. E. and J. B. Whitney (1975): "A likelihood analysis of the linear calibration problem", Technometrics, 17, no. 4, 463-471.

Misquitta, P. and F.H. Ruymgaart (200*): Some results on nonparametric calibration, Communications in Statistics - Theory and Methods, (To appear)

Osborne, C. (1991): "Statistical calibration: a review", International Statistical Review, 59, 309-336.

Pepper, M. P. G. (1973): "A calibration of instruments with non-random errors", T Technometrics, 15, 587-599.

Scheffé, Henry (1973): "A statistical theory of calibration", Annals of Statistics, 1, 1-37.

Srivastava, V. K. and N. Singh (1989): "Small disturbance asymptotic theory for linear calibration estimators", Technometrics, 31 189-192. 
Tallis, G. M. (1969): "Note on a calibration problem", Biometrika, 56, 505-508.

Williams, E.J. (1969) : A note on regression methods in calibration, Technometrics, 11, 189-192.

Williford, W. O., Michael C. Carter and Janet E. Field (1979): "A further look at the Bayesian approach to calibration", Journal of Statistical Computation and Simulation, 9 no. 1, 47-67. 